\title{
CLUZH at VarDial GDI 2017: Testing a Variety of Machine Learning Tools for the Classification of Swiss German Dialects
}

\author{
Simon Clematide \\ Institute of Computational Linguistics \\ University of Zurich \\ simon.clematidedcl.uzh.ch
}

\author{
Peter Makarov \\ Institute of Computational Linguistics \\ University of Zurich \\ makarov@cl.uzh.ch
}

\begin{abstract}
Our submissions for the GDI 2017 Shared Task are the results from three different types of classifiers: Naïve Bayes, Conditional Random Fields (CRF), and Support Vector Machine (SVM). Our CRF-based run achieves a weighted F1 score of $65 \%$ (third rank) being beaten by the best system by $0.9 \%$. Measured by classification accuracy, our ensemble run (Naïve Bayes, CRF, SVM) reaches 67\% (second rank) being $1 \%$ lower than the best system. We also describe our experiments with Recurrent Neural Network (RNN) architectures. Since they performed worse than our nonneural approaches we did not include them in the submission.
\end{abstract}

\section{Introduction}

The goal of our participation in the newly introduced German Dialect Identification (GDI) Shared Task of the VarDial Workshop 2017 (Zampieri et al., 2017) was to quickly test how far we could get on this classification problem using standard machine learning techniques (as only closed runs were allowed for this task).

The task is to predict the correct Swiss German dialect for manually transcribed utterances (Samardzic et al., 2016). ${ }^{1}$ The Dieth transcription (Dieth, 1986) - developed in the 1930s in Switzerland-is not a scholarly phonetic transcription system. It is designed to be applicable by laymen to all Swiss German dialects and uses the Standard German alphabet and a few optional diacritics.

In this task, the number of possible Swiss German dialects is limited to four main varieties: the

\footnotetext{
${ }^{1}$ Since the text segments are transcribed speech, with a slight abuse of terminology, we shall refer to them as utterances.
}

dialects spoken in the cantons of Basel (BS), Bern (BE), Lucerne (LU), and Zurich (ZH).

The four approaches that we have worked on for this task are: i) a powerful baseline that uses an off-the-shelf Naïve Bayes classifier trained on bags of character n-gram features; ii) an unconventional yet effective application of a CRF classifier to sequence classification-the system performing best on the official test set among all our runs; iii) a majority-vote ensemble of the Naïve Bayes, CRF and SVM systems; and iv) an RNN character-sequence classifier trained on augmented data, which however has not been included in our final submission. ${ }^{2}$

\section{Related Work}

Scherrer and Rambow (2010) describe dialect identification approaches to written Swiss German. To distinguish among six dialects, they experiment with a word n-gram model. Additionally, they attempt word-based identification by turning Standard German words into their dialectal forms according to hand-written transfer rules. They discuss the linguistic aspects of the problem and difficulties in predicting for the multitude and continuum of Swiss German dialects.

Most of our final submission, except probably Run 2, is an application of well-established techniques for text classification (Sebastiani, 2002). We use regularized linear classifiers on a bag-ofcharacter-n-grams representations of utterances. Despite its conceptual simplicity, this recipe produces state-of-the-art results on language identification tasks (Malmasi et al., 2016) and is particularly easy to implement given the wide variety of readily available tools for feature extraction and classification. Having this as a baseline, we

\footnotetext{
${ }^{2}$ Our code is available at https://github.com/ simon-clematide/GDI-task-2017.
} 


\begin{tabular}{|r|rl|rl|rr|rr|r|}
\hline & BE & BS & LU & ZH & Total \\
\hline Training Set & 3889 & 0.27 & 3411 & 0.24 & 3214 & 0.22 & 3964 & 0.27 & 14478 \\
Test Set & 906 & 0.25 & 939 & 0.26 & 916 & 0.25 & 877 & 0.24 & 3638 \\
\hline Difference & & $-2 \%$ & & $+2 \%$ & & $+3 \%$ & & $-3 \%$ & \\
\hline \hline Training 4+ & 3260 & 0.26 & 2974 & 0.24 & 2865 & 0.23 & 3327 & 0.27 & 12426 \\
\hline
\end{tabular}

Table 1: Distribution of classes in the training and test sets of the GDI task. Row "Training 4+" shows the effect of removing sentences with less than 4 tokens on the training set composition.

\begin{tabular}{|r|rrrrrrrrrr|}
\hline Tokens & 1 & 2 & 3 & 4 & 5 & 6 & 7 & 8 & 9 & $10+$ \\
\hline Training & 360 & 731 & 961 & 1244 & 1416 & 1491 & 1428 & 1317 & 1125 & 4405 \\
Rel. & $2 \%$ & $5 \%$ & $7 \%$ & $9 \%$ & $10 \%$ & $10 \%$ & $10 \%$ & $9 \%$ & $8 \%$ & $30 \%$ \\
\hline Test & & & & 495 & 530 & 465 & 450 & 368 & 320 & 1010 \\
Rel. & & & & $14 \%$ & $15 \%$ & $13 \%$ & $12 \%$ & $10 \%$ & $9 \%$ & $28 \%$ \\
\hline
\end{tabular}

Table 2: Distribution of numbers of tokens per utterance in the training and test sets of the GDI task.

focus on experimenting with CRFs and charactersequence neural network classifiers. Zhang et al. (2015) achieve competitive results on characterlevel document classification tasks with Convolutional Neural Networks (CNNs). Word-level RNNs have been applied to a variety of text classification tasks (Carrier and Cho, 2014). Xiao and Cho (2016) present an efficient character-level RNN document classifier.

\section{Data and Methodology}

In this section, we first describe the training and test data sets. Second, we detail the methods that we apply in our runs as well as report the results of post-submission experiments using RNNs.

\subsection{Properties of the Data}

As Table 1 shows, the GDI training data set has roughly balanced classes (a maximum of \pm 3 percentage points away from a uniform distribution). The official test set is slightly better balanced (a maximum of \pm 1 percentage points away from a uniform distribution). However, the data sets do not have the same minority/majority classes.

Another noticeable difference between the training and test data is the presence of short utterances. The training set has 2,052 utterances (14\%) which consist of only one, two or three words. This contrasts with the test set, whose utterances contain four or more words. Predicting the dialect of a short utterance is much harder than predicting the dialect of a long one. We systematically drop very short utterances from the training data in order to compensate for the differences between the data sets ${ }^{3}$ and to reduce the noise.

The data only contain lowercase characters. Due to the variability in the dialects, many of the 14,065 word types appear only once $(9,372)$, twice $(2,032)$, or three times (929). This extreme Zipfian distribution makes it hard to build reliable statistics for prediction.

\subsection{Our Methods}

All our methods except the RNNs use character $\mathrm{n}$-gram features derived from separate words.

\subsubsection{Run 1: Naïve Bayes}

Run 1 is our baseline, which has proven hard to beat. For the final submission, we drop from the training set short noisy utterances and substitute character combinations for characters with complex diacritics (e.g. "ï2" for "ǜ") and single characters for the common digraph "ch" and trigraph "sch". All one-character words are dropped. We represent each utterance with a bag of character ngrams, ranging from bigrams to six-grams. This set-up produces the highest average validation ac-

\footnotetext{
${ }^{3}$ This violates the default assumption in machine learning scenarios "that training and test data are independently and identically (iid) drawn from the same distribution. When the distributions on training and test set do not match, we are facing sample selection bias or covariate shift" (Huang et al., 2007). Different unsupervised domain adaptation techniques have been developed in order to mitigate this problem, e.g. instance weighting (Jiang and Zhai, 2007). A very simple weighting schema consists in assigning a weight of zero to short utterances, i.e. removing them. Two reviewers had the opinion that it is a methodological problem to adapt the models to the evidence in the test set and that one is not supposed to look at the test set at all. Ultimately, it is a question of the task guidelines whether unsupervised domain adaptation is considered legitimate or not.
} 

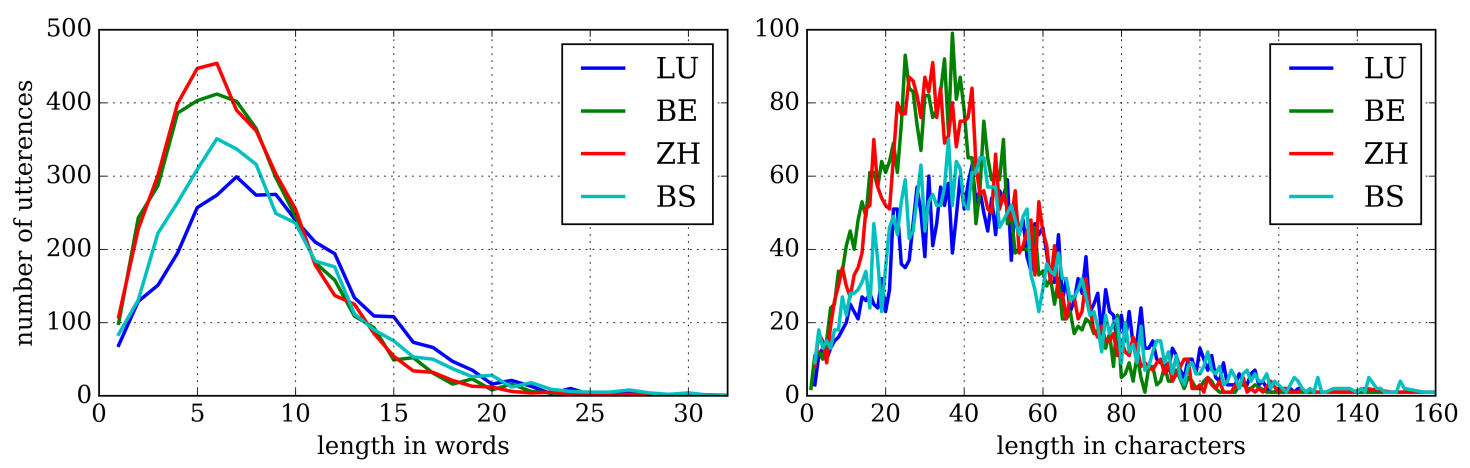

Figure 1: Per-dialect distribution of numbers of tokens and characters per utterance in the training set.

curacy among competing configurations (e.g. differing in n-gram ranges). We use the scikit-learn machine learning library (Pedregosa et al., 2011) to implement the entire pipeline. We fit a Naïve Bayes classifier with add-one smoothing.

\subsubsection{Run 2: CRF}

For Run 2, we use wapiti (Lavergne et al., 2010), an efficient off-the-shelf linear-chain CRF sequence classifier (Sutton and McCallum, 2012). Each word of an utterance is treated as a tagged item in a sequence and the utterance classification task is cast as a sequence classification of all items. For instance, the utterance "jaa ich han ja" with sequence label $\mathrm{ZH}$ is turned into a verticalized format corresponding to " $\mathrm{jaa} / \mathrm{ZH}$ ich/ZH han/ZH $\mathrm{ja} / \mathrm{ZH} "$.

The motivation behind this approach is that a single word is often ambiguous, however, we know a priori by the definition of the task that all words in an utterance must have the same class. Therefore, we rely on the machinery of CRFs to adjust the weights of the word features in the exponential model during training in such a way that sequences get optimally and homogeneously classified. Indeed, the predicted sequence of classification tags within one utterance is always consistent, and we take the class of the first word as the class of the whole utterance.

The features for the CRF are built from individual words. We experimented with different replacement rules for the diacritics, but in the end just applied two phonetically motivated replacements ("sch" and "ch") before feature extraction.

We use 4 types of features for the representation of a token:

WD The word form using our two replacements.
PS Concatenations of the prefix and suffix of each word (from 1 to 3 characters depending on the length of the word).

NG Character n-grams (from 1 to 6 characters). Before extracting the n-grams, we prefix each word with an "A" and suffix it with a "Z" in order to distinguish $\mathrm{n}$-grams at word boundaries from n-grams within a word.

$\mathrm{CV}$ Word shapes selecting or mapping character classes for consonants and vowels. Specifically, feature types $\mathrm{V}$ and $\mathrm{C}$ contain all vowels and consonants of a word in the order of appearance. Feature types Cs and Vs contain the sets of all consonants and vowels, respectively. Feature types VV and CC contain the word shape where either all vowels or all consonants get masked with a "C" or a "V". Feature type CCVV masks all characters with a "C" or a "V".

b Each word also has a so-called bigram output feature that encodes the transition probability of class labels. This ensures that the system learns to predict sequences with only homogeneous class labels. The unigram output feature "u", which encodes the global distribution of class labels, was not useful, however.

CRF tools like wapiti allow each feature to be used as evidence only for the class of the current token (feature prefix "u:") or the class of the preceding and/or current token (feature prefix "*:."). ${ }^{4}$ For the GDI task, we only use "u:" features. Thus, for a word like "vernoo" (en: heard), the following features are extracted:

\footnotetext{
${ }^{4}$ See Lavergne et al. (2010) for technical details.
} 


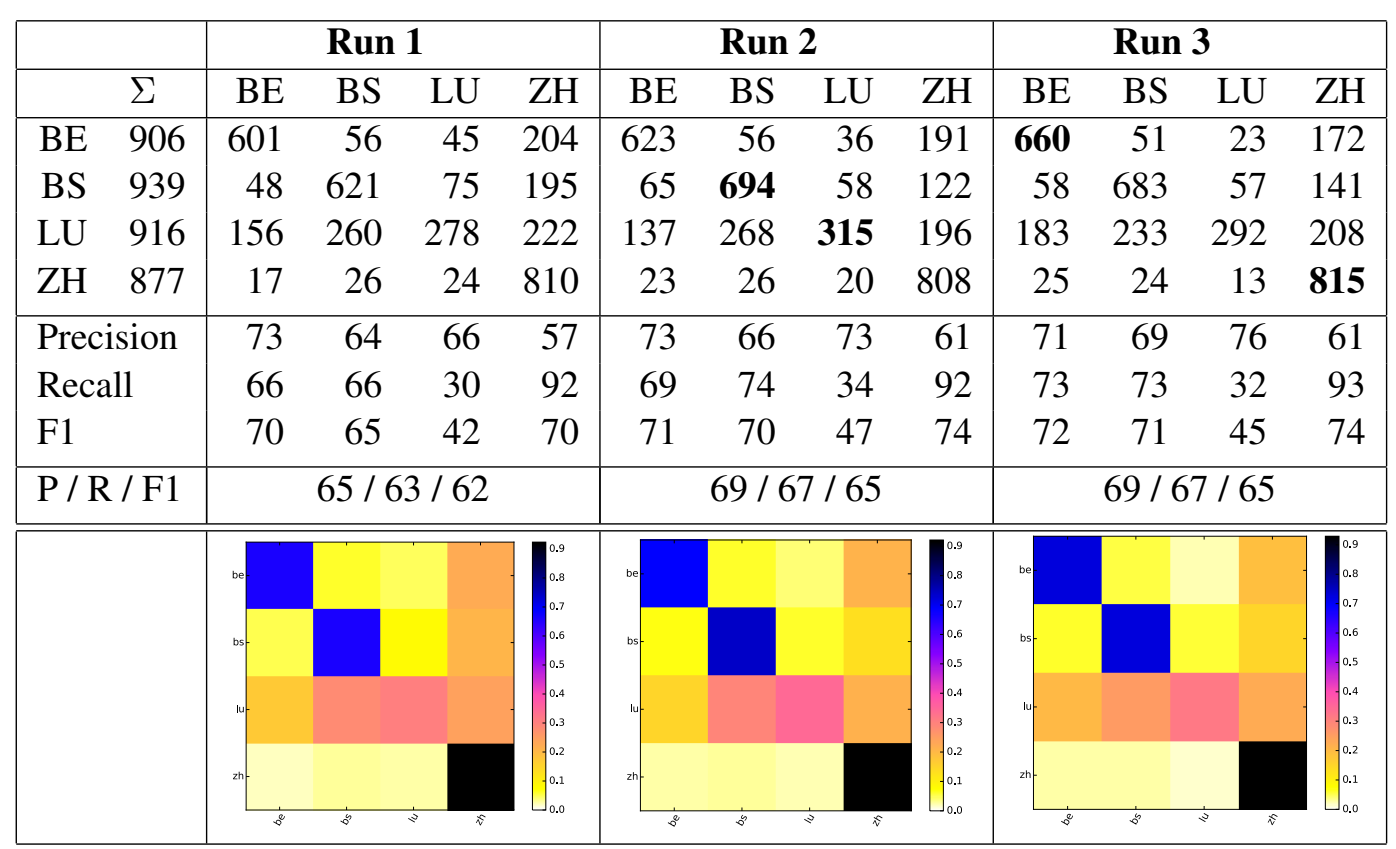

Table 4: Confusion matrices and result breakdown for our official GDI runs. Rows are true labels, columns are predicted labels.

\begin{tabular}{|rccc|}
\hline Run & Accuracy & F1 (macro) & F1 (weighted) \\
\hline Baseline & 25.80 & & \\
\hline 1 & 63.50 & 61.65 & 61.56 \\
2 & 67.07 & $\mathbf{6 5 . 3 8}$ & $\mathbf{6 5 . 3 1}$ \\
3 & $\mathbf{6 7 . 3 4}$ & 65.34 & 65.27 \\
\hline
\end{tabular}

Table 5: Official results for the GDI task. The baseline predicts the majority class. For all classes, F1 (micro) is the same as accuracy.

pletely (in this case, one-word and two-word utterances). As a result of this data augmentation, the training data for the internal system evaluation have grown by almost a quarter (from 11,726 to 15,340 utterances).

All neural-network implementation is done using high-level structures of the keras neural networks library (Chollet, 2015). For training the models, we use the Root Mean Square Propagation (RMSProp) algorithm (Tieleman and Hinton, 2012), a variant of Stochastic Gradient Descent, with default hyper-parameters suggested by the library. We use Dropout (Srivastava et al., 2014) for regularization. We train for at least 100 epochs and at most 300 epochs.

\section{Results}

\subsection{Official Results}

Table 5 shows the official results of our submitted runs. Run 3 has the best accuracy among our runs, but is slightly worse on the macro-averaged F1 score and the weighted F1 score (see Zampieri et al. (2017) for further information on the evaluation metrics). The performance in absolute numbers is much lower than expected from cross-validation.

\subsection{Internal Evaluation}

Table 6 shows average validation scores of the systems featured in our submissions. We retrain the systems with the same hyper-parameter settings as in the submissions. The ensemble performs best followed closely by the baseline system of Run 1. To compare the systems with the bestperforming LSTM from the post-submission experiments, we set aside a stratified sample of one tenth the size of the training data as an internal evaluation set. Again, we retrain the models on the remaining data with the same hyper-parameter settings. Since these hyper-parameter values have been found to produce the best performance on the entire training data, internal evaluation set results are potentially biased upward for all the systems but the LSTM. 


\begin{tabular}{|l|ccc|ccc|}
\hline & \multicolumn{3}{|c}{ Cross-validation results } & \multicolumn{3}{c|}{ Internal evaluation set results } \\
\hline Run & Accuracy & F1 (macro) & F1 (weighted) & Accuracy & F1 (macro) & F1 (weighted) \\
\hline 1 & $85.10(0.82)$ & $84.99(0.82)$ & $85.10(0.82)$ & 85.43 & 85.36 & 85.44 \\
2 & $83.96(0.68)$ & $83.87(0.70)$ & $83.93(0.69)$ & 85.01 & 85.02 & 85.01 \\
3 & $\mathbf{8 5 . 7 0}(\mathbf{0 . 5 9})$ & $\mathbf{8 5 . 5 7}(\mathbf{0 . 6 0})$ & $\mathbf{8 5 . 6 8}(\mathbf{0 . 6 0})$ & $\mathbf{8 5 . 5 0}$ & $\mathbf{8 5 . 4 2}$ & $\mathbf{8 5 . 5 0}$ \\
SVM & $82.46(0.59)$ & $82.36(0.64)$ & $82.43(0.61)$ & 82.39 & 82.36 & 82.39 \\
\hline LSTM & - & - & - & 83.49 & 83.30 & 83.46 \\
\hline
\end{tabular}

Table 6: System comparison: Results for ten-fold stratified cross-validation and performance on an internal evaluation set. Cross validation results: We report mean scores across the folds and indicate standard deviations in parentheses. The SVM is a model from the ensemble of Run 3.

\begin{tabular}{|c|c|c|c|c|c|c|c|c|}
\hline \multicolumn{3}{|c|}{ Model configuration } & \multicolumn{3}{|c|}{ Development set results } & \multicolumn{3}{|c|}{ Internal evaluation set results } \\
\hline $\begin{array}{l}\text { data } \\
\text { aug. }\end{array}$ & $\begin{array}{l}\text { char. } \\
\text { repl. }\end{array}$ & $\begin{array}{l}\text { char. } \\
\text { emb. }\end{array}$ & Accuracy & F1 (macro) & F1 (weighted) & Accuracy & F1 (macro) & F1 (weighted) \\
\hline- & - & - & 81.75 & 81.57 & 81.65 & 81.15 & 80.79 & 80.99 \\
\hline- & - & + & 81.52 & 81.35 & 81.51 & 81.98 & 81.90 & 81.96 \\
\hline - & + & - & 82.75 & 82.50 & 82.69 & 82.39 & 82.23 & 82.34 \\
\hline - & + & + & 80.83 & 80.60 & 80.74 & 79.90 & 79.71 & 79.84 \\
\hline+ & - & - & 81.60 & 81.42 & 81.53 & 83.22 & 83.08 & 83.18 \\
\hline+ & - & + & 82.82 & 82.66 & 82.78 & 82.60 & 82.52 & 82.59 \\
\hline+ & + & - & 82.52 & 82.35 & 82.52 & 83.49 & 83.30 & 83.46 \\
\hline+ & + & + & 82.59 & 82.43 & 82.56 & 82.04 & 81.91 & 82.00 \\
\hline
\end{tabular}

Table 7: Comparison of RNN sequence classifiers.

\section{Discussion}

$\mathrm{ZH}$ clearly dominates in terms of recall in all our runs (Table 4). The recognition rates for $\mathrm{ZH}, \mathrm{BE}$, and BS are fine (around 70\% F1) in our official runs. However, the F1 score for LU is much lower (around 45\%) due to severe recall problems. The numbers show that the recognition of LU suffers from more frequent predictions in favor of $\mathrm{ZH}$ and BS. This behavior fits the empirical distribution of the classes from the training set (short sentences removed) as shown in Table 1 where $27 \%$ of all sequences are $\mathrm{ZH}$, but only $23 \% \mathrm{LU}$. As the problem may also lie in the data, it would be interesting to see whether all the systems participating in the shared task exhibit this bias.

The results on the official test data (Table 5) are unexpectedly lower than our cross-validation estimates from the training data $(67 \%$ accuracy instead of about $88 \%$ with short sequences removed). Clearly, the training and test sets have not been consistently sampled from the same distribution.

The Naïve Bayes classifier of Run 1 has been exceptionally strong on same-domain data. Interestingly, it suffers worse compared to other systems from differently sampled data.
According to our observation during training, CRFs seem to run a bit into convergence problems. Therefore, one might try to systematically build more varying models (for instance, by bootstrap sampling and randomly selected subsets of extracted features) in order to have a broader ensemble system. Another line of work that we could not complete due to time restrictions is the integration of a word prediction model into the CRF system based on character-level CNNs (Xiao and Cho, 2016). Our expectation would be that convolution filters might be better at learning relevant character-level representations for estimating the label probability for a given word.

We have struggled to produce strong results with RNNs. By the submission deadline, no model had performed on a par with our nonneural systems. Table 7 presents the results of our post-submission experiments. Data augmentation brings about impressive gains of $0.7 \%$ on a development set and $1.5 \%$ on the internal evaluation set, on average across the three metrics. Character replacements largely hurt performance: On average, we see a drop of $0.2 \%$ on the development set and $0.9 \%$ on the internal evaluation set. The effects of a character embedding layer cancel out across the development and internal evaluation sets $(-0.2 \%$ 
and $+0.3 \%$, respectively). On the other hand, models with a character embedding layer and/or character replacements are faster to reach higher accuracy levels. Just like with other models, short utterances pose the largest difficulty, and performance goes up with utterance length. Overall, using slow-to-train neural models on this task has not paid off: Blazingly fast linear classifiers achieve very strong results, and so time is better spent on looking for good features.

\section{Conclusion}

We show that a character n-gram-based Naïve Bayes approach gives a very strong baseline for the classification of transcribed Swiss German dialects, especially when test and training sets are drawn from the same distribution. The CRFbased approach works better for the official test set (ranked third by weighted F1 score among all the submitted GDI runs). The official test set is clearly sampled differently from the training set. Given a rather large performance difference of $4.5 \%$ between the Naïve Bayes and the CRF, we suspect that the CRF-based approach has generalized better than the Naïve Bayes. In terms of accuracy, an ensemble approach using Naïve Bayes, CRF, and linear SVM gives the best results of our runs and ranks second among all GDI runs.

\section{Acknowledgement}

We would like to thank three anonymous reviewers for their helpful comments. Peter Makarov is supported by European Research Council Grant No. 338875.

\section{References}

Pierre Luc Carrier and Kyunghyun Cho. 2014. LSTM networks for sentiment analysis. Deep Learning Tutorials.

François Chollet. 2015. Keras. https: / / github. $\mathrm{com} / \mathrm{f}$ chollet/keras.

Eugen Dieth. 1986. Schwyzertütschi Dialäktschrift: Dieth-Schreibung. Lebendige Mundart. Sauerländer, Aarau etc. 2. Aufl. / bearb. und hrsg. von Christian Schmid-Cadalbert (1. Aufl. 1938).

Alex Graves and Jürgen Schmidhuber. 2005. Framewise phoneme classification with bidirectional LSTM and other neural network architectures. Neural Networks.

Sepp Hochreiter and Jürgen Schmidhuber. 1997. Long short-term memory. Neural computation.
J. Huang, A. Smola, A. Gretton, K. Borgwardt, and B. Schoelkopf. 2007. Correcting sample selection bias by unlabeled data. In B. Schoelkopf, J. Platt, and T. Hoffman, editors, Advances in Neural Information Processing Systems 19, pages 601-608. MIT Press, Cambridge, MA.

Jing Jiang and ChengXiang Zhai. 2007. Instance weighting for domain adaptation in NLP. In Proceedings of the 45th Annual Meeting of the Association of Computational Linguistics, pages 264-271. Association for Computational Linguistics.

Thomas Lavergne, Olivier Cappé, and François Yvon. 2010. Practical very large scale CRFs. In Proceedings the 48th Annual Meeting of the Association for Computational Linguistics (ACL). Association for Computational Linguistics.

D. C. Liu and J. Nocedal. 1989. On the limited memory BFGS method for large scale optimization. Math. Program.

Shervin Malmasi, Marcos Zampieri, Nikola Ljubešić, Preslav Nakov, Ahmed Ali, and Jörg Tiedemann. 2016. Discriminating between similar languages and arabic dialect identification: A report on the third dsl shared task. In Proceedings of the 3rd Workshop on Language Technology for Closely Related Languages, Varieties and Dialects (VarDial), Osaka, Japan.

F. Pedregosa, G. Varoquaux, A. Gramfort, V. Michel, B. Thirion, O. Grisel, M. Blondel, P. Prettenhofer, R. Weiss, V. Dubourg, J. Vanderplas, A. Passos, D. Cournapeau, M. Brucher, M. Perrot, and E. Duchesnay. 2011. Scikit-learn: Machine learning in Python. Journal of Machine Learning Research.

Tanja Samardzic, Yves Scherrer, and Elvira Glaser. 2016. ArchiMob - A corpus of spoken Swiss German. In Proceedings of the Language Resources and Evaluation (LREC), pages 4061-4066, Portoroz, Slovenia).

Yves Scherrer and Owen Rambow. 2010. Word-based dialect identification with georeferenced rules. In Proceedings of the 2010 Conference on Empirical Methods in Natural Language Processing. Association for Computational Linguistics.

Fabrizio Sebastiani. 2002. Machine learning in automated text categorization. ACM computing surveys (CSUR).

Nitish Srivastava, Geoffrey E Hinton, Alex Krizhevsky, Ilya Sutskever, and Ruslan Salakhutdinov. 2014. Dropout: a simple way to prevent neural networks from overfitting. Journal of Machine Learning Research.

Charles A. Sutton and Andrew McCallum. 2012. An introduction to conditional random fields. Foundations and Trends in Machine Learning. 
Tijmen Tieleman and Geoffrey Hinton. 2012. Lecture 6.5-rmsprop: Divide the gradient by a running average of its recent magnitude.

Yijun Xiao and Kyunghyun Cho. 2016. Efficient character-level document classification by combining convolution and recurrent layers. CoRR.

Marcos Zampieri, Shervin Malmasi, Nikola Ljubešić, Preslav Nakov, Ahmed Ali, Jörg Tiedemann, Yves Scherrer, and Noëmi Aepli. 2017. Findings of the VarDial Evaluation Campaign 2017. In Proceedings of the Fourth Workshop on NLP for Similar Languages, Varieties and Dialects (VarDial), Valencia, Spain.

Xiang Zhang, Junbo Zhao, and Yann LeCun. 2015. Character-level convolutional networks for text classification. In Advances in neural information processing systems.

Hui Zou and Trevor Hastie. 2005. Regularization and variable selection via the elastic net. Journal of the Royal Statistical Society: Series B (Statistical Methodology). 